\title{
Analysis of Trends in Mortality in Patients with Lymphoepithelial Carcinoma of the Head and Neck
}

\author{
Hector Picon' \\ Achuta Kumar Guddati iD ${ }^{2}$ \\ 'Medical College of Georgia, Augusta \\ University, Augusta, GA, 30909, USA; \\ ${ }^{2}$ Division of Hematology/Oncology, \\ Georgia Cancer Center, Augusta \\ University, Augusta, GA, 30909, USA
}

Background: Squamous cell carcinoma is the most common subtype of malignancy found in patients with head and neck malignancy. There are other rare subtypes which are not adequately reported in medical literature. Lymphoepithelial carcinoma consists of lymphocytic infiltration in a background of undifferentiated carcinoma. They are most often seen in salivary glands but can also be found in other structures of the head and neck region. This analysis reports the nation-wide mortality of patients diagnosed with lymphoepithelial carcinoma of the head and neck.

Methods: Data were extracted from the Surveillance, Epidemiology, and End Results (SEER) Database from the years 2000 to 2014. Incidence-based mortality for all stages was queried and results were grouped by gender and race (Caucasian/White, African American/Black, American Indian/Alaskan native and Asian/Pacific Islander). Paired T-test was used to determine statistically significance difference between various subgroups.

Results: Incidence-based mortality has been improving for African American/Black patients and has been worsening for Caucasian/White, American Indian/Alaskan native and Asian/Pacific Islander for the period of 2000 to 2014 . The differences in mortality trends were statistically different $(\mathrm{P}<0.05)$. The highest mortality rate per 1000 patients was seen in Asian/Pacific Islander population, followed by African American/Black, American Indian/Alaskan native and the least mortality was noted in Caucasian/White patients. When a similar analysis with linearized trend lines on gender was conducted, only African American/Black males and Asian/Pacific Islander females showed an improving trend in mortality. The sample size was a major limitation of this study (Caucasian/White - 134, African American/Black - 30, American Indian/Alaskan native -5 and Asian/Pacific Islander - 87).

Conclusion: Lymphoepithelial carcinoma is a rare subtype of head and neck malignancies whose incidence-based mortality showed a worsening trend. This study showed significant race and gender disparity amongst patients with lymphoepithelial carcinoma. Due to its rarity, this subtype warrants further study, especially with regards to its etiology, clinical course and cure rates.

Keywords: lymphoepithelial carcinoma, mortality, race, age

\section{Introduction}

Lymphoepithelial carcinoma (LEC) is a rare type of undifferentiated carcinoma of the major salivary glands characterized by atypical, malignant epithelial cells integrated with lymphoid infiltrate. ${ }^{1,2}$ It occurs primarily in the parotid gland but can also be found in the submandibular gland and nasopharynx, as well as other areas of the head and neck, and it accounts for less than $0.4 \%$ of malignant salivary gland tumors. ${ }^{3,4}$ Clinically, LEC often presents as painless mass and is sometimes
Correspondence: Achuta Kumar Guddat Division of Hematology/Oncology, Georgia Cancer Center, Augusta

University, Augusta, GA, 30912, USA

Tel + I 312-404-8928

Email aguddati@augusta.edu 
associated with facial nerve palsy or cervical lymphadenopathy. ${ }^{17}$ The age of the patient at presentation is variable but the ages of 20 to 60 are the most common, with a median age of 40 years. ${ }^{17}$

LEC is known to have a similar histological appearance to undifferentiated nasopharyngeal carcinoma, likely due to the common developmental roots of the organs involved. ${ }^{16}$ The cancers exhibit obvious lymphocytic infiltrate of the stroma, with small lymphocytes and plasma cells being the most common. ${ }^{3}$ The infiltrate surrounds islands of large, irregularly shaped epithelial cells with round or oval vesicular nuclei and at least one prominent nucleolus. ${ }^{3,16}$ However, an important difference is that the vast majority of patients with nasopharyngeal carcinoma also have Epstein-Barr virus (EBV), whereas EBV is associated with only certain races for LEC. ${ }^{16}$ The Inuits of the Arctic region and the southern Chinese are two populations that experience higher incidence of LEC with EBV, but this has not been observed in Caucasians or other non-endemic populations. ${ }^{5,6}$

Some researchers have attempted to further study LEC in non-endemic areas. For example, one study using the National Cancer Database, a dataset that is exclusive to the United States, found that incidence of LEC was highest in Caucasians 50-70 years of age, with no significant difference between genders. ${ }^{7}$ However, the change in mortality of LEC over time has yet to be studied. It is important to understand the prognosis of LEC and the role that demographic variables play in that prognosis in order to direct efforts at reducing cancer disparities. Our study examines these variables by querying a dataset for incidence-based mortality. The data is obtained from death certificates of cancer patients in the United States, and it does have limitations. For example, it may not take into account recent advances in cancer therapies because the deaths occurred before the effect of the therapies could be seen. This method also prevents a deeper analysis of the data by grouping cases by certain characteristics such as onset of disease, age at diagnosis, etc. These limitations aside, incidence-based mortality is generally accepted as a reliable way of measuring progress against cancer. ${ }^{18}$ Our study uses data from the National Cancer Institute's Surveillance, Epidemiology, and End Results (SEER) Database to examine the rates of incidence-based mortality from the years 2000 to 2014 amongst racial groups and genders. Our goal is to validate previous research on the topic and provide new insight as to the influence of demographics in order to improve care for patients diagnosed with LEC.

\section{Methods}

\section{Data Source}

The Surveillance, Epidemiology, and End Results (SEER) database of the National Cancer Institute was used to identify patients with LEC. This database contains information on cancer incidence, mortality, and patient demographics from approximately $34.6 \%$ of the population of the United States, and coverage varies by race $(31.9 \%$ of Whites, $30.0 \%$ of African-Americans, $44 \%$ of Hispanics, 49.3\% of American Indians and Alaskan Natives, 57.5\% of Asians, and $68.5 \%$ of Hawaiians/Pacific Islanders).

\section{Study Population}

The study included patients that were diagnosed with LEC according to the World Health Organization's criteria in the third edition of the International Classification of Diseases for Oncology (ICD-O-3). Patients of all ages and all stages of disease progression were included, provided that the diagnosis occurred between 2000 and 2014 . Patient information concerning race and gender was obtained for every case, and cases that had unknown race or gender were excluded from the study. Cases were separated into four racial groups: Caucasian/White, African American/Black, American Indian/Alaskan Native, and Asian/Pacific Islander.

\section{Outcomes}

Incidence-based mortality for LEC was calculated for race and gender. Rates are reported as number of cases per 1000 and were age-adjusted to the 2000 US standard population. Incidence-based mortality rates was defined as number of LEC deaths among cases diagnosed over person-time at risk among people in SEER areas. ${ }^{8,9}$

\section{Statistical Analysis}

SEER*stat software was used to calculate incidence-based mortality. $T$-tests were used to calculate $\mathrm{P}$ values for differences identified among the groups listed above. $\mathrm{P}$ values were only considered statistically significant when less than 0.05 .

\section{Results}

Changes in incidence-based mortality are displayed in Figure 1. The negative slope for the African American/ Black regression line shows that mortality has been decreasing for this population, whereas the other three racial groups show an increase in mortality over the analyzed time period. 


\section{Linear Regression of Changes in Mortality Rate from 2000-2014}

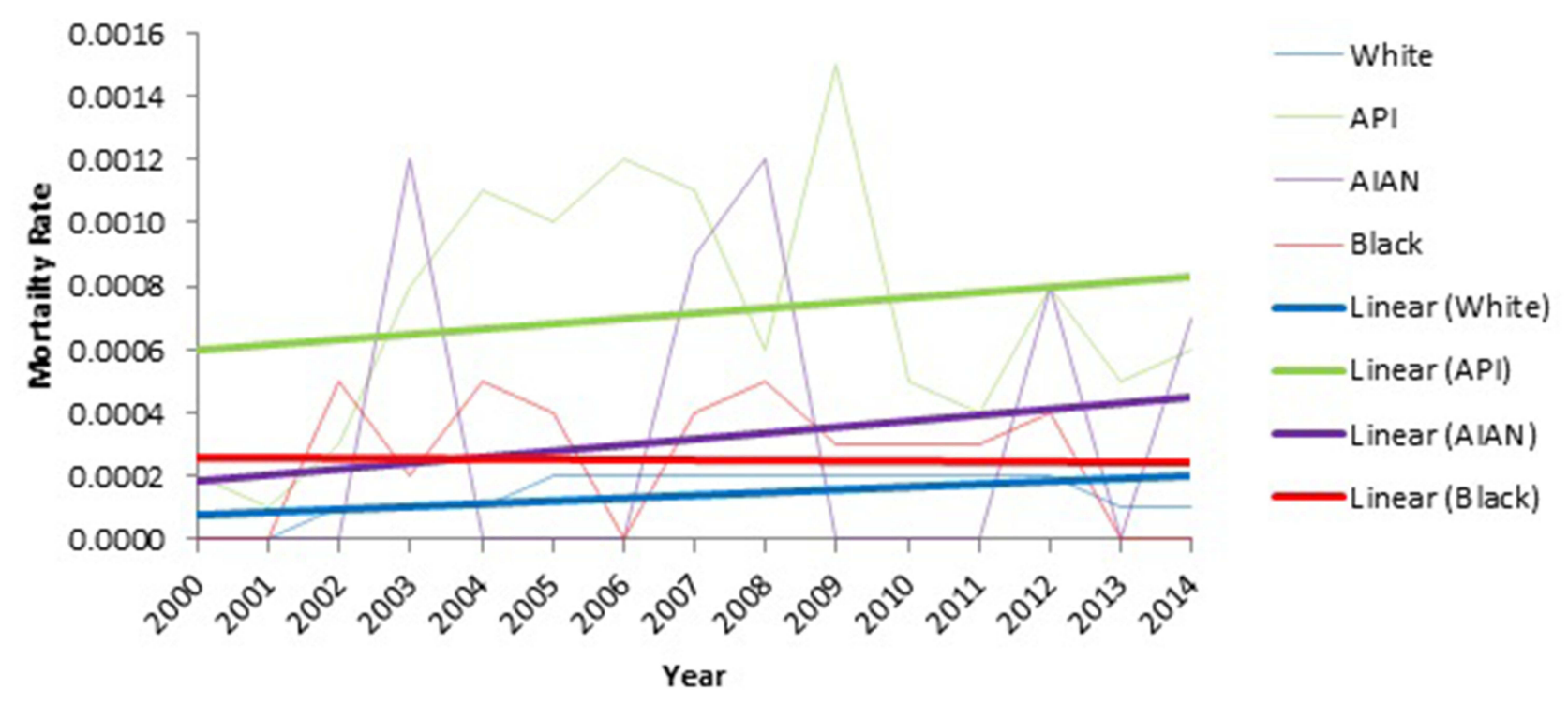

Figure I Changes in incidence-based mortality rates for LEC based on race [Caucasian, African American, Asian/Pacific Islander (API) and American Indian/Alaskan Native (AIAN)]. The faded lines are the data points, while the corresponding linear regression are shown in dark lines.

The observed differences were statistically significant for $\mathrm{P}<0.05$. The above data was summed into total cases diagnosed and subsequently averaged into mortality rates per racial group for the studied time period (Table 1). The highest mortality rates are seen in Asians/Pacific Islanders, followed by American Indians/Alaskan Natives, African Americans/Blacks, and Caucasian/Whites, respectively. The limited sample size of LEC cases, indicated by the second column, should also be noted.

Further classification of the data by gender within racial groups is shown in Figure 2. Of the subclassifications, the only groups that showed a decreasing trend in

Table I Aggregate Incidence-Based Mortality of LEC per 1000 Patients from the Years 2000 to 2014

\begin{tabular}{|l|c|c|c|}
\hline Race & $\begin{array}{c}\text { LEC } \\
\text { Cases }\end{array}$ & Population & $\begin{array}{c}\text { Rate } \\
\text { (per 1000) }\end{array}$ \\
\hline Caucasian/ White & 134 & $916,519,947$ & 0.00015 \\
\hline African American/ Black & 30 & $135,847,855$ & 0.00022 \\
\hline $\begin{array}{l}\text { American Indian/ Alaskan } \\
\text { Native }\end{array}$ & 5 & $18,718,615$ & 0.00027 \\
\hline Asian/Pacific Islander & 87 & $121,397,292$ & $0.00072^{*}$ \\
\hline
\end{tabular}

Note: $*_{p}<0.05$ denotes statistically significant difference. incidence-based mortality were African American/Black males and Asian/Pacific Islander females, again denoted by the negative slope of the trend lines. All other subclassifications showed an increasing trend in mortality.

\section{Discussion}

Race is an important, known factor in cancer disparities. Previous studies have thoroughly documented the disparities seen between racial groups. For example, African Americans/Blacks experienced a 15\% higher cancer death rate than whites in 2014. ${ }^{11}$ Asians/Pacific Islanders and American Indians/Alaskan Natives experience lower rates of death and cancer incidence when compared to Caucasians/Whites for 4 of the most common cancers, but the reverse is true when considering cancers of infectious agents. ${ }^{11}$ In head and neck cancers specifically, African Americans/Blacks have been shown to have higher rates of short-term mortality, lower rates of overall survival, and higher recurrence of cancer. ${ }^{12,13}$ Thus, the influence of race warrants further studies in specific cancers in order to work toward eliminating disparities.

Cancer disparities are also seen between genders. Men have a slightly higher chance of being diagnosed with invasive cancer than women, at $40.8 \%$ versus $37.5 \%$, the reasons for the higher incidence is largely unknown. ${ }^{11,14}$ 
A

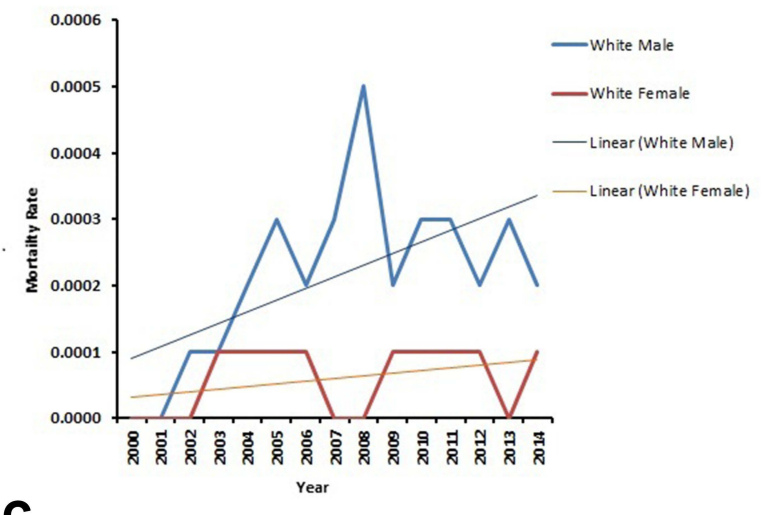

C

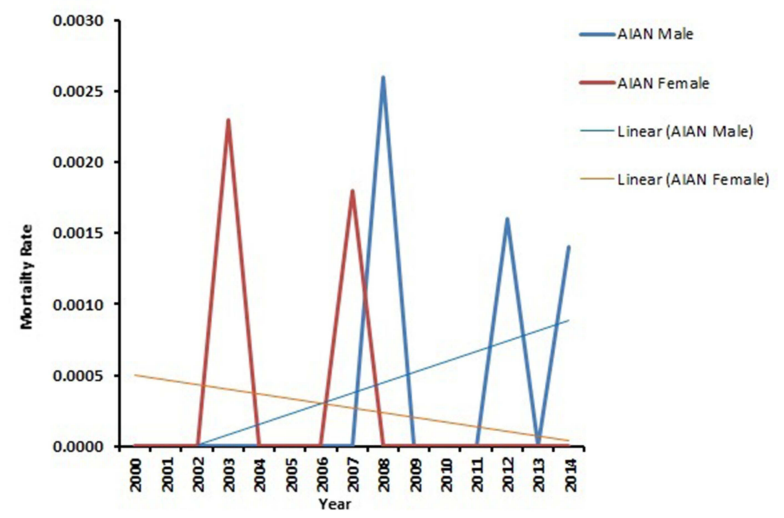

B

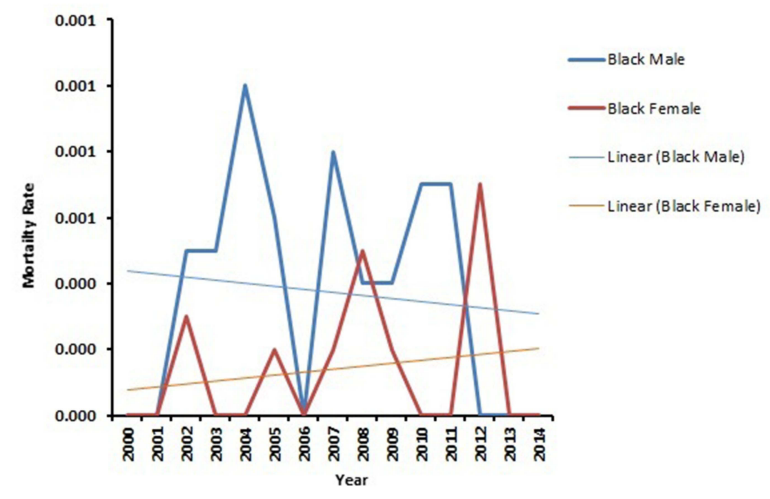

D

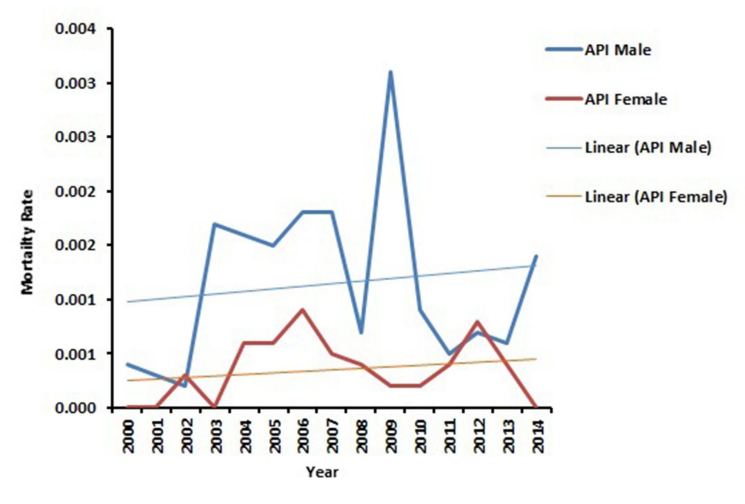

Figure 2 Changes in incidence-based mortality between genders of racial groups. The faded lines are the data points, while the corresponding linear regression is shown more darkly. The panels are separated by race for (A) Caucasian/White, (B) African American/Black, (C) American Indian/Alaskan Native (AIAN), and (D) Asian/Pacific Islander (API).

Disparities can even be seen in specific cancers of the head and neck, such as larynx and esophageal cancer, where men experience 4-fold higher rates of incidence and death. ${ }^{11}$ Thyroid cancer, on the other hand, shows higher rates of incidence in women. ${ }^{15}$ These statistics indicate that gender should be considered as an important variable when analyzing head and neck cancer outcomes.

In our study, disparities in incidence-based mortality of LEC were evident between different racial groups, with Caucasians showing a statistically significant increase in mortality from 2000 to 2014. Further, linearized trend analysis on gender showed that all groups except African American/Black males and Asian/Pacific Islander females have experienced a statistically significant increase in incidence-based mortality. This is different from a similar study by Zhan et al where no disparity in 5- and 10-year survival for LEC of the salivary glands was seen when comparing genders or when comparing Caucasians versus other races. ${ }^{7}$ This difference may be attributed to the fact that our study takes all types of LEC into account rather than just those of the salivary gland. Other possible explanations include that the studies analyzed slightly different time periods and used different databases, as both of these factors could skew the results.

It should also be noted that we did not look for associations between LEC and Epstein-Barr virus. This is because previous studies have found that the association is dependent upon racial and geographical factors specific to the endemic areas of the Arctic region and southern China. ${ }^{6,10}$ Neither of these populations were expected to be heavily represented in the SEER database, but the association between LEC and Epstein-Barr virus should be considered in studies that do include these populations.

There are many inherent limitations that must be taken into account. The most significant limitation is sample size, especially with regards to American Indians/Alaskan Natives. As noted in Table 1, there were only 5 reported cases of LEC in this population from 2000 to 2014. Such a small sample size makes it difficult to draw conclusions from linearized trend analysis, but we maintain that the available data should still be analyzed. Additionally, because we examined incidence-based mortality only, important 
variables such as age at diagnosis and stage of disease at diagnosis were left out. These can be indicators of disease prognosis but were not available in the SEER database.

\section{Conclusion}

This study analyzes the influence of race and gender on incidence-based mortality of lymphoepithelial carcinoma in patients represented in the SEER database from 2000 to 2014. Most of the racial groups analyzed, with the exception of African Americans/Blacks, showed an increase in mortality over the years. Most of the gender groups analyzed also showed an increase in mortality, with Asian/ Pacific Islander females and African American/Black males being the exceptions. This study is "hypothesis generating" and hence a starting point for a larger and a more detailed study. Future studies should examine the etiology, clinical course, and cure rates of LEC in order to further investigate this rare but important cancer.

\section{Ethics Statement}

This study is based on a publicly available national database which has de-identified patient data with no patient contact or intervention.

\section{Acknowledgments}

Preliminary results shown in the study were presented as an abstract at ASCO 2019 and may be found at https:// ascopubs.org/action/showCitFormats?doi=10.1200/JCO. 2019.37.15 suppl.e17583. This manuscript is not under consideration in any other journal. The authors declare that there was no funding for this study. All authors have read the manuscript and agree to the content.

\section{Funding}

There is no funding to report.

\section{Disclosure}

The authors report no conflicts of interest in this work.

\section{References}

1. Wang CP, Chang YL, Ko JY, Lou PJ, Yeh CF, Sheen TS. Lymphoepithelial carcinoma versus large cell undifferentiated carcinoma of the major salivary glands. Cancer. 2004;101(9):2020-2027. doi:10.1002/cncr.20614
2. Ambrosio MR, Mastrogiulio MG, Barone A, et al. Lymphoepitheliallike carcinoma of the parotid gland: a case report and a brief review of the western literature. Diagn Pathol. 2013;8:115. doi:10.1186/ 1746-1596-8-115

3. Schneider M, Rizzardi C. Lymphoepithelial carcinoma of the parotid glands and its relationship with benign lymphoepithelial lesions. Arch Pathol Lab Med. 2008;132(2):278-282. doi:10.1043/1543-2165(2008)132[278:Lcotpg]2.0.Co;2

4. Kim YJ, Hong HS, Jeong SH, Lee EH, Jung MJ. Lymphoepithelial carcinoma of the salivary glands. Medicine. 2017;96(7):e6115. doi:10.1097/md.0000000000006115

5. Manganaris A, Patakiouta F, Xirou P, Manganaris T. Lymphoepithelial carcinoma of the parotid gland: is an association with Epstein-Barr virus possible in non-endemic areas? Int J Oral Maxillofac Surg. 2007;36 (6):556-559. doi:10.1016/j.ijom.2006.12.012

6. Kuo T, Hsueh C. Lymphoepithelioma-like salivary gland carcinoma in Taiwan: a clinicopathological study of nine cases demonstrating a strong association with Epstein-Barr virus. Histopathology. 1997;31 (1):75-82. doi:10.1046/j.1365-2559.1997.5830814.x

7. Zhan KY, Nicolli EA, Khaja SF, Day TA. Lymphoepithelial carcinoma of the major salivary glands: predictors of survival in a non-endemic region. Oral Oncol. 2016;52:24-29. doi:10.1016/j. oraloncology.2015.10.019

8. Chu KC, Miller BA, Feuer EJ, Hankey BF. A method for partitioning cancer mortality trends by factors associated with diagnosis: an application to female breast cancer. J Clin Epidemiol. 1994;47 (12):1451-1461. doi:10.1016/0895-4356(94)90089-2

9. Saad AM, Gad MM, Al-Husseini MJ, Ruhban IA, Sonbol MB, Ho TH. Trends in renal-cell carcinoma incidence and mortality in the United States in the last 2 decades: a SEER-Based Study. Clin Genitourin Cancer. 2019;17(1):46-57.e5. doi:10.1016/j. clgc.2018.10.002

10. Tsang WYW. Lymphoepithelial carcinoma. Pathol Genet. 2005:251-252. Available from:: https://ci.nii.ac.jp/naid/ 10030271433/en/. Accessed September 22, 2021.

11. Siegel RL, Miller KD, Jemal A. Cancer statistics, 2017. CA Cancer J Clin. 2017;67(1):7-30. doi:10.3322/caac.21387

12. Gaubatz ME, Bukatko AR, Simpson MC, et al. Racial and socioeconomic disparities associated with 90-day mortality among patients with head and neck cancer in the United States. Oral Oncol. 2019;89:95-101. doi:10.1016/j.oraloncology.2018.12.023

13. Naghavi AO, Echevarria MI, Strom TJ, et al. Treatment delays, race, and outcomes in head and neck cancer. Cancer Epidemiol. 2016;45:18-25. doi:10.1016/j.canep.2016.09.0051

14. Wiren S, Haggstrom C, Ulmer H, et al. Pooled cohort study on height and risk of cancer and cancer death. Cancer Causes Control. 2014;25 (2):151-159. doi:10.1007/s10552-013-0317-7

15. Aschebrook-Kilfoy B, Ward MH, Sabra MM, Devesa SS. Thyroid cancer incidence patterns in the United States by histologic type, 1992-2006. Thyroid. 2011;21(2):125-134. doi:10.1089/ thy. 2010.0021

16. Hsu YC, Lu HF, Huang CC, Hsu RF, Su CY. Malignant lymphoepithelial lesions of the salivary gland. Otolaryngol Head Neck Surg. 2006;134(4):661-666. doi:10.1016/j.otohns.2005.10.034

17. Ellis GL, Auclair PL. Tumors of the Salivary Glands. Washington, DC: Armed Forces Institute of Pathology; 1996:311-318, 411-413. Atlas of Tumor Pathology; 3rd series, fascicle 17.

18. Extramural Committee to Assess Measures of Progress Against Cancer. Measurement of progress against cancer. J Natl Cancer Inst. 1990;82(10):825-835. doi:10.1093/jnci/82.10.825 


\section{Publish your work in this journal}

The International Journal of General Medicine is an international, peer-reviewed open-access journal that focuses on general and internal medicine, pathogenesis, epidemiology, diagnosis, monitoring and treatment protocols. The journal is characterized by the rapid reporting of reviews, original research and clinical studies across all disease areas. The manuscript management system is completely online and includes a very quick and fair peer-review system, which is all easy to use. Visit http://www.dovepress.com/ testimonials.php to read real quotes from published authors. 